\title{
Analyse in situ des savoirs mobilisés par un professeur d'éducation physique et sportive dans l'interaction didactique
}

\section{Fabienne Brière-Guenoun et Chantal Amade-Escot}

Cette étude sintéresse aux savoirs mobilisés par un enseignant d'éducation physique et sportive (EPS) en situation ordinaire d'enseignement de la gymnastique au secondaire I. Les savoirs de l'enseignant sont appréhendés à partir d'une analyse de ses gestes de "conception des dispositifs d'étude» et «d'aide à l'étude». La méthodologie employée pour accéder au caractère situé des savoirs s'appuie sur un couplage articulant des enregistrements filmés et des entretiens de type "auto-confrontations». Les résultats montrent la mobilisation de savoirs variés orientés par des valeurs éducatives, traduisant les convictions propres de l'enseignant et au-delà ses appartenances aux diverses institutions traversées.

\section{Introduction}

Cet article concerne l'identification des savoirs mobilisés par le professeur d'éducation physique et sportive (EPS) dans sa pratique quotidienne et la compréhension des processus de leur activation ${ }^{1}$. De nombreux travaux en sciences de l'éducation distinguent plusieurs types de savoirs professionnels: des savoirs théoriques et d'action (Barbier, 1996), des savoirs d'expérience et ceux acquis grâce à la formation (Tardif, Lessard \& Lahaye, 1991), des connaissances pédagogiques et celles sur la matière enseignée (Shulman, 1987). Ces visions dichotomiques tendent aujourd'hui à être remplacées par une conception plus intégrative de ces différents savoirs, considérés comme encapsulés dans les pratiques (Perrenoud, Altet, Lessard \& Paquay, 2008).

La spécificité des matières scolaires et les contraintes du système didactique influencent aussi les savoirs mobilisés in situ. S'intéresser aux savoirs mobilisés par les enseignants en contexte s'inscrit ainsi dans un projet plus vaste qui relève de la mise au jour de l'épistémologie pratique des professeurs comme déterminant «du» didactique (Sensevy, 2007). Dans ce cadre, notre travail d'analyse se situe au carrefour de la théorie de l'action conjointe en didactique (TACD) (Schubauer-Leoni, 2008; Sensevy \& Mercier, 2007) et de la théorie anthropologique 
du didactique (TAD) (Chevallard, 1992). Il vise l'étude des dispositifs proposés par un professeur d'EPS pour enseigner le "franchissement par redressement au saut de cheval $»^{2}$ lors d'un cycle de gymnastique en classe de sixième.

\section{Cadre théorique: Savoirs de référence du professeur et direction d'étude}

Loption retenue est celle d'une analyse ascendante de la transposition didactique (Schubauer-Leoni \& Leutenegger, 2005) seule àmême de permettre l'accès aux savoirs mobilisés par l'enseignant en contexte. Néanmoins, pour démêler dans le syncrétisme de la pratique observée ce à quoi attribuer le statut de «savoir», nous avons emprunté à Chevallard (1999) la notion de "praxéologie» associée selon cet auteur à celle de gestes professionnels.

Une analyse ascendante des savoirs à partir de l'analyse des gestes du professeur

Dans cette recherche, nous étudions les gestes du professeur (Chevallard, 1999) en vue d'inférer, selon une démarche de type ascendant, les savoirs qu'il mobilise dans l'interaction didactique. En d'autres termes, l'analyse repose sur ce qui se passe dans la classe mais sans perdre de vue que la légitimité de cette réalisation tient aux références externes (Schubauer-Leoni, 2008) de types savants, experts ou personnelles (Johsua, 1996).

Par ailleurs, les savoirs du professeur s'expriment à travers ses gestes de direction d'étude, définis dans la TAD comme d'une part des "gestes de conception et d'organisation des dispositifs d'étude" qui concernent la détermination des tâches d'apprentissage en lien avec les «organisations (ou praxéologies) mathématiques» 3 à étudier et d'autre part des "gestes d'aide à l'étude», consistant à conduire la reconstruction (ou transposition) dans la classe de cette organisation (Chevallard, 1997). La posture anthropologique adoptée dans la TAD souligne que ces gestes dépendent de l'activité d'apprentissage effective des élèves. Ce point autorise, selon nous, des perspectives d'analyse de l'action du professeur compatibles avec les approches ascendantes de la TACD.

\section{Les gestes du professeur liés à la mise en jeu de «praxéologies»}

Pour Chevallard, toute pratique, et donc celle du professeur, peut être analysée en tant que système de tâches, c'est-à-dire d'activités relativement bien circonscrites dont la réalisation est permise par la maitrise d'une «technique» ou manière de faire (1997, p. 38). Ce système plus ou moins intégré de types de tâches et de techniques, nommé «bloc practico-technique», peut être assimilé à ce que l'on nomme couramment un "savoir-faire» (Chevallard, 1999). A ce "système tâchestechniques» est associé un "système théorico-technologique» au sein duquel la 
technologie désigne un discours rationnel (le logos) sur la pratique dont l'objet est de justifier, d'expliquer, voire de produire de nouvelles techniques. Ce discours technologique nécessite à son tour un niveau supérieur de justification- explication-production, celui de la théorie, dont le caractère est abstrait. L'auteur donne un statut de "savoir» à cet ensemble théorico-technologique. Le terme de "praxéologie» désigne ainsi une organisation bi-polaire reliant le logos à la praxis. Cette notion (déclinée en praxéologies disciplinaire ou didactique) est pertinente pour appréhender les savoirs de l'enseignant car ces derniers sont encapsulés dans l'action et en conséquence peu dissociables des pratiques. Dans cette recherche, nous avons fait un usage méthodologique de la notion de praxéologie pour inférer à partir des gestes de direction d'étude ce qui relève des savoirs mobilisés par le professeur.

\section{Les savoirs inférés à partir de la reconstruction de deux types des praxéologies}

Toute activité d'enseignement met en jeu des "praxéologies disciplinaires» (ici gymniques) qui renvoient à la réalité disciplinaire mise à l'étude et des "praxéologies didactiques", qui concernent la manière dont peut se réaliser l'étude de cette réalité (Brière-Guenoun, 2005; Chevallard, 1999). Ce système conceptuel permet d'analyser les pratiques et leurs ancrages théoriques tant du point de vue des choix disciplinaires effectués que des modalités d'enseignement proposées in situ. Au sein des praxéologies didactiques, les techniques didactiques du professeur, dont Sensevy (2002) souligne le caractère communicationnel et polyfonctionnel, sont envisagées selon le point de vue du «modèle de l'action du professeur» (Sensevy, Mercier \& Schubauer-Leoni, 2000), qui repose sur l'identification de quatre structures de l'action dans la relation didactique: définir, réguler, dévoluer, institutionnaliser. Ces structures fondamentales se déclinent en types de tâches puis en "types de gestes et de techniques d'action» (SchubauerLeoni, Leutenegger, Ligozat \& Fluckiger, 2007, p. 59) qui renvoient aux manières dont le professeur construit le jeu conjoint avec les élèves. Ces techniques rendent compte des façons dont professeur et élèves font évoluer le temps de l'enseignement et de l'apprentissage (chronogenèse), dont se construisent les places respectives de l'élève et de l'enseignant (topogenèse) et dont évolue le milieu au fil des interactions (mésogenèse) en vue d'établir un système partagé de significations (Sensevy, 2007).

C'est à partir de ces descripteurs (structures de l'action didactique et triplet des genèses) que nous reconstruisons les praxéologies gymniques et didactiques au principe de l'action de l'enseignant. Ainsi, en nous appuyant sur les fondements de la théorie de l'action conjointe, nous faisons l'hypothèse que les gestes du professeur peuvent être décrits en termes de praxéologies, ce qui permet d'inférer les savoirs mobilisés dans l'action. 
Une typologie des savoirs adaptée aux sciences et techniques des activités physiques et sportives (STAPS) Pour Johsua, la transposition didactique dans le cadre scolaire relève d'une «transposition de savoirs» impliquant «des modèles de pratiques, qui s'en séparent qualitativement» (1996, p. 65). Pour l'auteur, ces modèles convoquent: i) des "savoirs savants», liés à des communautés à qui la société a délégué le droit de dire le vrai; ii) des "savoirs experts», développés par des communautés plus res-treintes au sein d'institutions non savantes; iii) des «savoirs personnels», propres à un seul ou quelques individus seulement. Nous avons considéré que les registres savants et experts participent au bloc théorico-technologique des praxéologies.

En EPS, les savoirs experts, représentés par des savoirs techniques ou «technologiques» (Bouthier \& Durey, 1994), occupent une place centrale dans les processus transpositifs. Pour rendre compte des savoirs mobilisés par le professeur dans l'action en EPS, nous avons spécifié les catégories de savoirs de Johsua (1996) en nous inspirant des distinctions établies par Léziart (1997) dans le champ des STAPS ainsi que des cadres implicites à l'œuvre dans l'architecture des cursus de cette filière universitaire ${ }^{4}$. Ainsi, les catégories adoptées dans cette étude distinguent: i) des savoirs scientifiques contributoires des sciences du sport qui relèvent des «savoirs savants» dès lors qu'ils sont légitimés par des communautés scientifiques; ii) des savoirs techniques issus des ouvrages spécialisés et des savoirs liés aux pratiques didactiques en usage tels que diffusés dans la littérature professionnelle des enseignants d'EPS, qui appartiennent au registre des «savoirs experts». Ces catégories, relativement perméables à leurs frontières, sont organisées sur un continuum.

Finalement, c'est en analysant les gestes professoraux mis en œuvre dans l'action conjointe que les praxéologies sont identifiées pour accéder par inférence aux savoirs du professeur et aux modalités de leur activation.

\section{Méthode}

Pour rendre compte des savoirs mobilisés par le professeur ayant fait l'objet de notre étude, nous avons observé les quatre premières séances du cycle ${ }^{5}$.

\section{Une configuration signifiante de traces}

La recherche s'inspire partiellement de l' «approche clinique et expérimentale du didactique ordinaire» (Schubauer-Leoni \& Leutenegger, 2002). Le terme de clinique renvoie à l'identification d'un certain nombre d'indices ou de signes dont les configurations permettront de rendre compte du fonctionnement didactique de la classe (Leutenegger, 2009). Postulant que pour caractériser les savoirs mobilisés par l'enseignant, il faut analyser comment celui-ci conçoit puis régule les dispositifs d'apprentissage au fil des interactions avec les élèves, nous n'avons pas, contrairement à la démarche classiquement proposée en didactique, introduit de 
perturbations majeures dans le fonctionnement naturel de la classe. Le chercheur s'est seulement assuré de l'existence dans le projet d'enseignement du professeur du thème d'étude "franchir par redressement au cheval», dont une analyse $a$ priori a été réalisée, sans pour cela orienter le choix des tâches proposées aux élèves. Nous avons retenu comme essentielle l'élaboration d'une configuration signifiante de traces rattachées à une double analyse «interne et externe» en vue d'établir, à travers leur «questionnement réciproque», «la distance nécessaire entre une construction de signes par le chercheur et ce qui appartient au terrain observé et fait sens pour les acteurs» (Schubauer-Leoni \& Leutenegger, 2002, p. 245).

Notre approche méthodologique est étroitement articulée avec la position théorique selon laquelle l'action didactique est le résultat du travail conjoint du professeur et des élèves (Sensevy, 2000), position déjà assumée par les premières recherches en didactique de l'EPS (Marsenach, 1991).

\section{Description et analyse du thème d'étude Un thème d'étude représentatif de l'activité gymnique}

Le franchissement par redressement s'organise autour de la coordination de deux envols décrits dans la figure 1. Le premier envol permet, en utilisant la vitesse acquise dans la course d'élan, de prendre appui avec les mains sur le cheval. Le deuxième envol, initié à partir de l'appui manuel, consiste à repousser le cheval pour se réceptionner en position verticale équilibrée. Ce thème d'étude est associé à un enchaînement d'actions, dont la figure 1 représente une illustration. La tâche générique présentée ici peut être modifiée en jouant sur l'aménagement matériel (type et hauteur de l'obstacle, surface d'impulsion, aire de réception, etc...) ou sur les formes de franchissement autorisées (saut groupé ou écart, franchissement sur le côté, etc...).

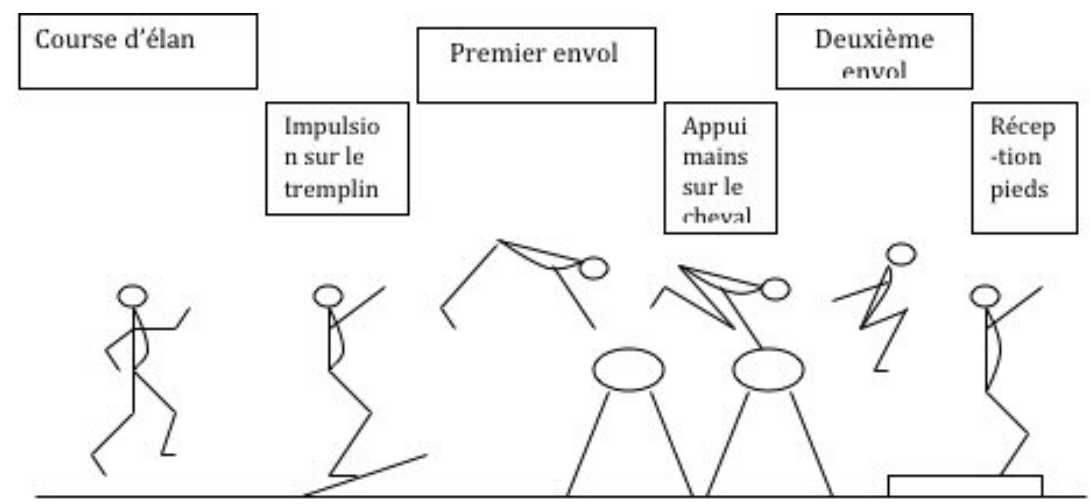

Figure 1: Schéma de la tâche générique mettant en jeu le franchissement par redressement. 


\section{Analyse a priori du thème d'étude et modèles technologiques en gymnastique} L'analyse a priori du thème d'étude ${ }^{6}$ réalisée par le chercheur didacticien permet de prévoir les conduites probables des élèves sur le dispositif en relation avec les objets de savoir sollicités. En nous appuyant sur les travaux de Robin (2003) portant sur l'identification des savoirs de référence développés par quatre leaders de théories gymniques scolaires, nous avons dégagé deux modélisations expertes appartenant respectivement au «registre technique» et au «registre des savoirs sur les pratiques didactiques en usage» 7 . Ainsi, l'analyse a priori $\mathrm{du}$ «franchissement par redressement au saut de cheval» peut être envisagée de deux façons selon que l'on se réfere à une technologie ${ }^{8}$ centrée sur les conditions formelles d'efficacité du geste définies d'un point de vue descriptif ou à une technologie valorisant la logique de l'action, en lien avec l'identification d'obstacles d'apprentissage.

Le premier type d'analyse s'inscrit dans la continuité des travaux de Carrasco (1975), qui définit les éléments gymniques d'un point de vue interne en relation avec une classification des actions impliquées (comme le gainage ou la répulsion des bras). Dans cette optique, Piard (1982) souligne l'existence de deux trajectoires étroitement liées (les premier et deuxième envols) entre lesquelles le contact des mains sur le cheval permet le changement d'orientation. Pour cet auteur, le franchissement par redressement se découpe en trois opérations (ou phases) fondamentales: la liaison course-appel, la liaison appel-appui et le franchissementréception. Selon Robin (2003), ce type de modélisation s'appuie sur des savoirs relevant du registre de la mécanique, de la psychologie piagétienne, et aussi, dans le cas de Piard, de l'anatomie fonctionnelle.

Le deuxième type d'analyse, qui repose sur les travaux de Goirand (1990, 1998), valorise les notions de risques et de virtuosité en relation avec les aspects émotionnels ainsi que les problèmes inhérents à la «référence culturelle nécessaire à la formulation des savoirs scolaires» (Robin, 2003, p.39). Goirand (1998) envisage trois étapes dans la construction du franchissement par redressement en fonction des logiques de l'approche (du cheval), de l'impulsion et de vol. Ces étapes se déclinent en conduites typiques marquant des niveaux de réalisation, dont la définition est articulée aux aspects fonctionnels et non descriptifs du geste. Selon cette logique, «le second envol est premier dans la genèse de la technique de franchissement» alors que «ce qui est habituellement appelé premier envol est génétiquement second» (Goirand, 1990, p.83). Pour Robin (2003), les analyses de Goirand s'appuient sur des références culturelles issues de la sociologie, de la psychologie, des neurosciences, de la biomécanique et «l'activité adaptative de l'élève gymnaste est au cœur des formalisations» (p.40) qu'il propose.

Ces deux technologies et les savoirs qui en relèvent constitueront un point de repère pour étudier les gestes effectifs de l'enseignant.

\section{Les participants à l'étude}

L'enseignant observé, appelé Damien, a neuf ans d'expérience professionnelle. Non spécialiste de l'activité, il a cependant pratiqué la gymnastique durant sa 
formation initiale universitaire en Sciences et Techniques des Activités Physiques et Sportives (STAPS). L'étude a porté sur ses interventions au cours d'un cycle de gymnastique avec une classe de sixième, dans un établissement classé en Zone d'éducation prioritaire (ZEP). La classe est constituée de 19 élèves (11 garçons et 8 filles) âgés de 11 à 14 ans, dont les compétences scolaires sont relativement hétérogènes et dont certains présentent des comportements de rejet de l'école.

\section{Recueil et traitement des données}

Le corpus principal est constitué par l'enregistrement filmé de toutes les séquences au cours desquelles le «franchissement par redressement» a été mis en place, associé à des Entretiens en Auto-Confrontations (EAC), réalisés à l'issue de chaque leçon observée. Les EAC sont ici spécifiés en regard des enjeux didactiques de la recherche à travers un guide de relance évolutif et souple, portant sur l'analyse des enjeux de savoir qu'attribue l'enseignant aux situations et régulations proposées (ou envisagées) ainsi que sur les façons dont il interprète les conduites des élèves. Destinés à centrer l'attention de l'enseignant sur l'action effective9, ces entretiens sont donc envisagés comme techniques d'accès contrôlé par le chercheur aux raisons de l'activation de certains savoirs. Ils ont le statut des «entretiens post» définis par Schubauer-Leoni et Leutenegger (2002). Comptetenu du caractère morphocinétique (Serre, 1984) de la gymnastique, le choix a été fait de prendre en compte le point de vue des élèves à travers leurs réponses et verbalisations in situ, sans recourir à des entretiens chercheur/élèves.

Un traitement séquentiel des différentes données a été opéré sur l'ensemble des quatre séances en délimitant des épisodes ${ }^{10}$ dans le flux des interactions. Les analyses mettent en relation des données relatives au pôle enseignant (recueillies lors des entretiens), des données concernant le savoir (analyse a priori et a posteriori des tâches à partir des traces filmées) et des données sur les élèves, leurs comportements et échanges verbaux avec l'enseignant dans les tâches réalisées à partir des enregistrements filmés ${ }^{11}$. Le traitement concomitant des données intrinsèques et extrinsèques pointe l'intérêt d'une remise en cause du «primat de l'intrinsèque» dans l'étude de l'activité professorale afin d'éviter le piège du réductionnisme qui, parce que présupposant que tout est dans le discours, réduit l'analyse de l'enseignement au subjectivisme de l'action professionnelle (AmadeEscot, 2005).

Ce corpus principal est complété par un corpus secondaire, composé d'un entretien ante-cycle centré sur les intentions de l'enseignant pour le cycle, d'un entretien post-cycle portant sur l'explicitation de ses cadres d'analyse et de traces écrites (projet de cycle).

\section{Méthode d'analyse}

En fonction des traces (épisodes vidéo retenus, entretiens ante-cycle ou post-cycle, projet de cycle), différents indices ont été retenus afin de renseigner par recoupement les praxéologies activées par l'enseignant. Les indices concernant les 
objets de savoir manipulés dans les dispositifs mis à l'étude, les interprétations de l'enseignant sur les conduites motrices des élèves (dans ses interventions verbales auprès des élèves en action et dans ses interprétations au cours des EAC), les modalités d'intervention ont permis de documenter les système tâches-techniques des praxéologies gymniques et d'en déduire les fondements théorico-technologiques. Les éléments pris en compte pour reconstruire les praxéologies didactiques relèvent de l'organisation spatio-temporelle des leçons, de la gestion des tâches et des techniques didactiques exploitées par l'enseignant pour conduire les séances.

Le traitement couplé des enregistrements filmés et des EAC, considéré comme une entité indissociable, est ensuite mis en relation avec les intentions didactiques de l'enseignant (entretien ante-cycle, traces écrites). L'entretien post-cycle, enfin, permet de confirmer ou d'infirmer les analyses relevant du croisement des corpus précédents. En résumé, le croisement des différentes traces permet d'inférer, à partir des fondements théorico-technologiques ainsi reconstruits, les savoirs mobilisés par l'enseignant.

\section{Analyse des données: Gestes de conception et de régulation des dispositifs d'apprentissage}

Pour présenter les résultats, nous exposons les intentions didactiques de l'enseignant avant de décrire ses gestes de conception, puis de régulation, des dispositifs d'apprentissage. Ce premier niveau d'analyse est développé en termes de fonctionnalité didactique en reprenant les catégories chronogénétiques, topogénétiques, mésogénétiques (Sensevy, Mercier \& Schubauer-Leoni, 2000), qui sont au cour des gestes de direction d'étude.

\section{Organisation du cycle et intentions de l'enseignant}

Le cycle est organisé autour de deux ateliers au sol ${ }^{12}$ orientés vers la production d'un enchaînement ${ }^{13}$ et de deux ateliers centrés sur la prise de risques: i) le salto avant avec trampoline (une rotation avant réalisée sans appui manuel au-dessus $\mathrm{du}$ sol) et ii) le franchissement par redressement au cheval, étudié dans cet article. Ce fonctionnement répond aux intentions didactiques de l'enseignant qui visent à "concilier une entrée dans l'activité sur le versant acrobatique correspondant à un désir d'agir en vue d'un plaisir immédiat propre aux élèves de sixième et sur le versant esthétique grâce à la production d'un enchaînement» (projet de cycle). Selon lui, "l'activité demande une réelle rigueur dans les apprentissages ainsi qu'une gestion de ses émotions et de son stress» et "favorise l'autonomie, le travail à deux (parade), l'entraide» (projet de cycle). Ces modalités organisationnelles marquent une certaine adhésion de la part de Damien au projet d'EPS, lui-même référé aux préconisations institutionnelles pour les ZEP. 
Gestes de conception des dispositifs d'apprentissage L'analyse des dispositifs d'apprentissage mis à l'étude nous renseigne sur les systèmes tâches/techniques des praxéologies gymniques et sur leurs fondements théorico-technologiques. Elle repose sur la confrontation d'analyses a priori des tâches, d'analyse des verbalisations de l'enseignant lors des épisodes concernant la présentation des tâches (corpus filmé) et dans les EAC.

Pour enseigner le thème d'étude "franchir par redressement», Damien met en place un dispositif unique, spécifié par des conduites de franchissement hiérarchisées. La tâche générique (voir figure 1) est alors façonnée comme un millefeuilles en plusieurs niveaux de réalisation. Le tableau 1, qui reprend la fiche mise en place par Damien au cours du cycle, décrit ces différents niveaux de tâches.

\section{Analyse a priori des tâches: spécificité des différents niveaux de franchissement} L'analyse a priori des tâches prescrites indique que: i) les niveaux 1, 2 et 4 correspondent à une augmentation progressive de la difficulté du point de vue des objets de savoir relatifs au premier envol; ii) le franchissement direct, c'est-à-dire sans arrêt sur le cheval, n'est proposé qu'à partir du troisième niveau; iii) seuls, les niveaux 4 impliquent la construction des objets de savoir propres au redressement en sollicitant une action active de blocage articulaire des épaules coordonnée à une fermeture des jambes entre les bras, dont l'enjeu est d'inverser le sens de la rotation (contre-rotation). La dimension affective de cette situation est liée à des enjeux mécaniques: le risque de basculer vers l'avant dépend de la capacité à bloquer (au niveau des épaules) la quantité de rotation lors de l'appui sur le cheval. Mais le blocage articulaire des épaules n'est justement pas nécessaire pour les franchissements de côté (niveaux $3 \mathrm{~A}$ et $3 \mathrm{~B}$ du tableau 1) qui, en autorisant le passage des jambes sur le côté (en dehors de l'axe de course), permettent à l'élève de contourner les problèmes posés par le redressement. On peut donc s'attendre à ce que certains élèves s'investissent et restent cantonnés dans les niveaux 3 sans pouvoir réellement construire les savoirs nécessaires à la réalisation des niveaux supérieurs. Nous considérons donc ces niveaux intermédiaires comme peu denses en savoirs (Schubauer-Leoni, 2008).

Ainsi, la hiérarchisation des niveaux s'inspire en partie des conduites typiques proposées par Goirand (1998), auxquelles elle rajoute les franchissements sur le côté (niveaux 3) et ne marque que partiellement l'évolution des enjeux de savoir associés au franchissement par redressement. La façon dont l'enseignant accompagnera l'évolution des élèves sur ces différents niveaux sera donc déterminante ${ }^{14}$.

\section{Analyse des enjeux de savoir définis par Damien dans la mise en place des dis- positifs proposés}

L'analyse conjointe des interventions de Damien lorsqu'il introduit les tâches (images filmées) et des verbalisations issues des EAC permet d'identifier les objets de savoirs qu'il retient dans la mise en place des niveaux de franchissement hiérarchisés. Des extraits de cette étude sont repris dans le tableau 2. 
Tableau 1: Les niveaux de franchissement hiérarchisés proposés par Damien

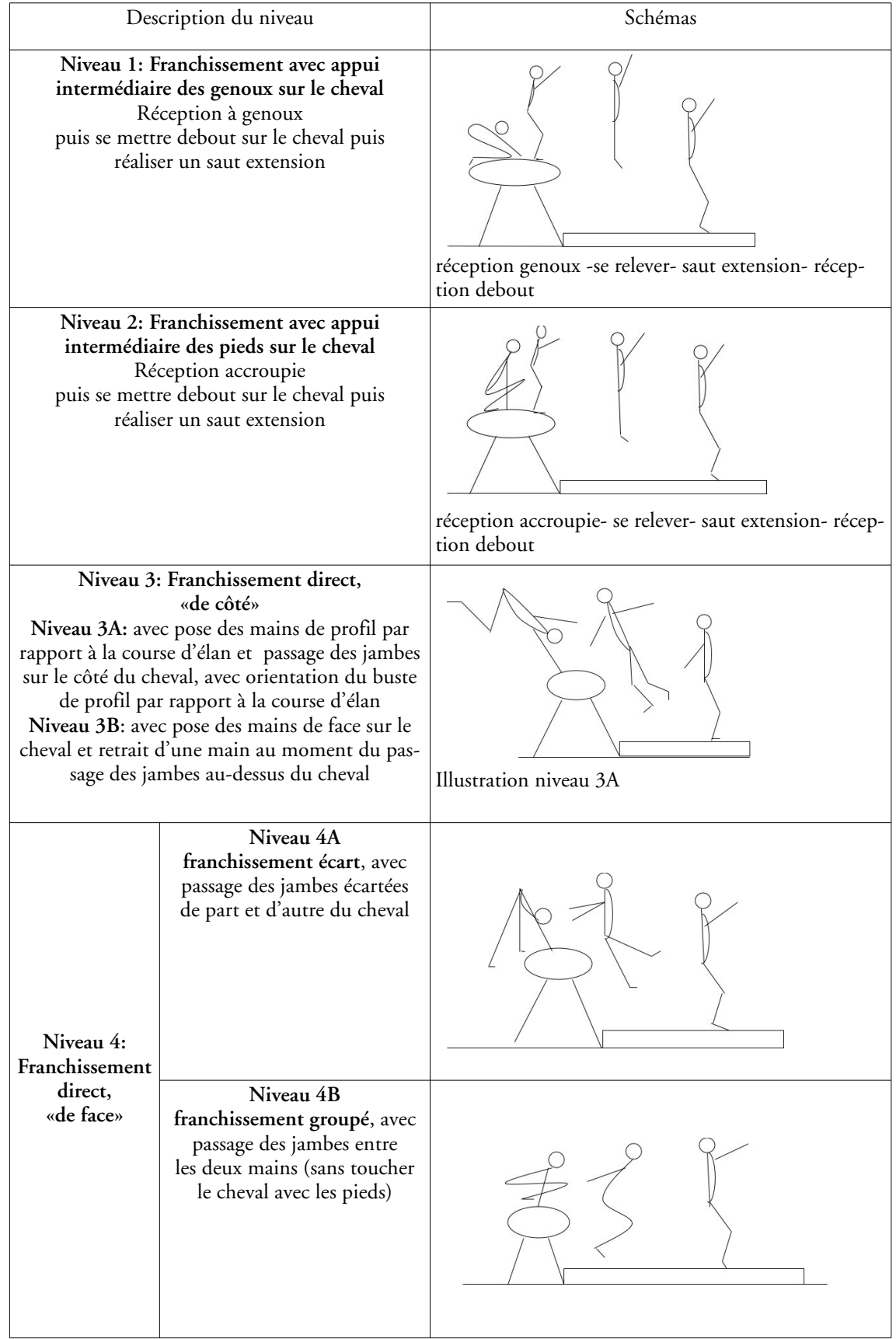


Tableau 2: Extraits relatifs à l'analyse des enjeux de savoirs envisagés par Damien, tirés des épisodes filmés, des EAC et de l'entretien ante.

\begin{tabular}{|c|c|}
\hline Objet de l'extrait & Extraits illustratifs \\
\hline $\begin{array}{l}\text { Identification des enjeux de savoir } \\
\text { pour classer les différents niveaux } \\
\text { de franchissement }\end{array}$ & $\begin{array}{l}\text { E1: «donc avec un niveau un où y'a un premier envol moyen, un } \\
\text { deuxième envol moyen et après une prise de recul du tramp, où } \\
\text { y'a un premier envol et un deuxième envol, donc écart ou groupé»" } \\
\text { (entretien ante-cycle) }\end{array}$ \\
\hline $\begin{array}{l}\text { Définition de l'impulsion lors de la } \\
\text { présentation des tâches }\end{array}$ & $\begin{array}{l}\text { E2: «percuter le tremplin pour monter puis franchir directement } \\
\text { sans poser les pieds sur le cheval, ou bien en posant les pieds» } \\
\text { (séance 2, épisode 3) } \\
\text { E3: «bien enfoncer le tremplin» (séance 2, épisode 4) }\end{array}$ \\
\hline \multirow[t]{2}{*}{$\begin{array}{l}\text { Définition du redressement lors de } \\
\text { la présentation des tâches }\end{array}$} & $\begin{array}{l}\text { E4: "repousser» et "d'être costand dans les épaules pour se ren- } \\
\text { voyer», "pour ne pas basculer vers l'avant» } \\
\text { (séance 2, épisode 3) }\end{array}$ \\
\hline & $\begin{array}{l}\text { E5: - «appuyer sur le tramp pour monter au- dessus du cheval et } \\
\text { appuyer sur le cheval pour repartir», } \\
\text { - «monter les fesses, s'appuyer sur le cheval et rebondir de nou- } \\
\text { veau», } \\
\text { - «toucher le cheval avec un appui bref des mains», } \\
\text { - "se redresser avec un appui bref et dynamique au niveau des } \\
\text { bras» (séance 4, épisode 4) }\end{array}$ \\
\hline $\begin{array}{l}\text { Définition des acquisitions nécessitées } \\
\text { par le redressement }\end{array}$ & $\begin{array}{l}\text { E6: «créer un transfert, un temps de bascule, un temps de ferme- } \\
\text { ture» et «se rebalancer après avoir posé les mains pour se redresser } \\
\text { et se remettre en situation équilibrée» } \\
\text { (séance 4, épisode 9) }\end{array}$ \\
\hline
\end{tabular}

Les axes de complexification de la tâche sont liés à des éléments de technicité gymnique comme l'impulsion, l'élévation du bassin au premier envol, l'existence d'un deuxième envol (extrait E1 du tableau 2) et se traduisent dans la détermination des formes de franchissement.

Les enjeux de savoirs considérés comme vifs par Damien sont spécifiés au fur et à mesure des séances par l'introduction progressive des différents niveaux de franchissement. Lors de la deuxième séance, Damien désigne les enjeux de savoirs liés à l'impulsion dans le tremplin (extraits E2 et E3 du tableau 2) qu'il met en relation avec les niveaux 1 et 2 . Dès la troisième séance, il veut amener tous les élèves à réaliser un franchissement direct, de côté (niveaux 3) ou de face (niveaux 4). Cet objectif, repris en quatrième séance, est relié au redressement, dont l'enseignant précise les acquisitions dans l'EAC (extraits E4 et E5 du tableau 2).

Nous considérons que la fiche des niveaux de franchissement représente un artefact permettant au professeur de gérer la chronogenèse par rapport aux enjeux de savoirs mis à l'étude. Dans ce contexte, pour définir les objets de savoirs, Damien se réfere à des descriptions relatives à l'analyse interne des actions caractérisant les deux envols, comme l'«impulsion jambes» ou la "répulsion des bras». Ce type de discours appartient à la technologie de Carrasco (1975), organisée autour de savoirs issus du registre technique. 


\section{Une chronogenèse subordonnée aux niveaux de franchissement}

L'avancée du temps didactique repose sur la construction de niveaux de franchissement pouvant être assimilés à «une désyncrétisation de formes gestuelles intermédiaires» (Marsenach, 1991), c'est-à-dire une élémentarisation du savoir visant à maintenir le sens global de l'activité tout en aménageant des étapes. Les niveaux de franchissement sont déterminés en regard des aspects fonctionnels du saut, en relation avec les dimensions émotionnelle et affective, conformément aux formalisations technologiques de Goirand (1990, 1998). La fiche des niveaux de franchissement délimite une situation de référence ou «situation ombilicale» (Chevallard, 1999), donnant l'occasion d'un travail de la technique au sens de Chevallard (1997).

A l'origine conçue par l'enseignant comme un outil pour mettre les élèves en projet, la hiérarchisation des niveaux devient, au fur et à mesure de l'avancée du cycle, un moyen de conduire les séances à travers l'identification d'objets d'apprentissage spécifiques prenant le statut de savoirs vifs au fil des séances.

\section{Gestes d'aide à l'étude}

L'analyse des gestes de régulation montre comment l'enseignant intervient in situ sur le dispositif en fonction des réponses des élèves et des contraintes de gestion de la classe. Les régulations sur le milieu didactique, défini comme «le système des objets (matériels, symboliques, langagiers) qui détermine les pratiques d'étude des savoirs» (Amade-Escot, 2007, p.120), concernent à la fois les interventions verbales de l'enseignant et les modifications opérés sur l'aménagement matériel des dispositifs. De manière générale, les régulations sont destinées à encourager les élèves, guider leurs réalisations et maintenir leur engagement dans la tâche. Elles restent ponctuelles en raison du positionnement de Damien, le plus souvent entre les deux ateliers à risques. Les variables utilisées pour réguler les tâches, peu nombreuses et conformes à celles exploitées dans les programmes ou dans les pratiques en usage, concernent l'aménagement de la réception, la forme de franchissement produite, l'existence ou non d'appuis intermédiaires sur le cheval et la distance tremplin-cheval.

\section{Une incitation à franchir directement}

Au cours de la deuxième séance, l'analyse conjointe des données filmées et de l'EAC illustre comment Damien intervient auprès d'un groupe d'élèves filles, dont certaines sont en difficulté. Après avoir installé un tapis devant et un autre derrière le cheval en vue de rassurer les élèves parce qu'il estime «qu'elles ont de l'appréhension» (EAC séance 2, épisode 4), il reste près du cheval pour accompagner leurs passages, comme retranscrit dans le tableau 3. 
Tableau 3: Extraits significatifs des interventions verbales de Damien, tirés de l'épisode 4 de la séance 2.

\begin{tabular}{|c|c|}
\hline Données d'enregistrement filmé & $\begin{array}{l}\text { Données d'entretien EAC } \\
\text { (post-séance) }\end{array}$ \\
\hline Retranscription des images filmées & Verbatim de l'entretien \\
\hline $\begin{array}{l}\text { Damien: Vous allez essayer de faire directement un appui, je } \\
\text { pars, je touche le cheval qu'avec les mains! }\end{array}$ & \multirow{7}{*}{$\begin{array}{l}\text { Chercheur: Là, les élèves ont décidé de } \\
\text { passer sur le côté. Cela est-il conforme à } \\
\text { tes objectifs ? } \\
\text { Damien: Dans l'immédiat, oui, parce que } \\
\text { je travaille surtout sur le franchissement } \\
\text { direct. Enfin, là, c'était surtout le côté se } \\
\text { rassurer puis franchir l'obstacle, je veux } \\
\text { pas dire quelle que soit la manière, mais } \\
\text { en posant appui mains, de côté ou pas. } \\
\text { Après, oui, euh, pour euh comment pour } \\
\text { amener le saut groupé ou le saut écart, il } \\
\text { va pas falloir renforcer beaucoup de fois } \\
\text { ce saut euh de côté parce que ça peut être } \\
\text { une limite. Maintenant, le saut de côté, } \\
\text { c'est le saut, je dirais, un peu naturel } \\
\text { qu'ils retrouvent et puis ça leur permet } \\
\text { quand même de travailler le ler envol et } \\
\text { l'élévation. Donc, je m'en sers. } \\
\text { Chercheur: Peux-tu expliquer pourquoi } \\
\text { ça leur permet? } \\
\text { Damien: Ben pour justement passer les } \\
\text { jambes sans toucher le cheval... C’est } \\
\text { plus rassurant. Enfin, je pense. C'est un } \\
\text { côté rassurant pour eux, euh, de passer les } \\
\text { jambes d'un côté, parce que s'ils se sont } \\
\text { pas suffisamment élevés, bon, ils vont } \\
\text { pouvoir se rattraper dans ce saut là sur les } \\
\text { genoux. Alors, bon, tu peux le retrouver } \\
\text { en saut groupé. Ils peuvent se rattraper } \\
\text { mais en saut écart, ils ne peuvent pas se } \\
\text { rattraper. }\end{array}$} \\
\hline $\begin{array}{l}\text { Alison: De côté ? } \\
\text { Damien: Oui, de côté, pensez à bien enfoncer le tramp! }\end{array}$ & \\
\hline $\begin{array}{l}\text { Alison passe (Damien est à la parade près du cheval) et réa- } \\
\text { lise un franchissement de type N3A avec élévation - } \\
\text { (franchissement de côté, en posant les mains de profil, avec } \\
\text { élévation du bassin en dessous de l'horizontale) } \\
\text { Damien: Enfonce bien le tremplin : on doit entendre le bruit! }\end{array}$ & \\
\hline $\begin{array}{l}\text { Sonia passe (Damien à la parade) et ne parvient pas à } \\
\text { monter sur le cheval } \\
\text { Damien: Il faut franchir sans toucher! } \\
\text { Sonia: J'y arrive pas! } \\
\text { Damien: Mais si! Il faut juste que tu coures un peu plus vite! }\end{array}$ & \\
\hline $\begin{array}{l}\text { Estelle passe (Damien à la parade près du cheval) et réalise } \\
\text { un franchissement de type N3B avec élévation - (fran- } \\
\text { chissement de côté, mains de face, avec élévation du bassin } \\
\text { en dessous de l'horizontale) } \\
\text { Damien: Oui, c'est ça! Pense à bien monter les fesses! }\end{array}$ & \\
\hline $\begin{array}{l}\text { Kelly passe (Damien à la parade près du cheval) et réalise } \\
\text { un franchissement de type N3B avec élévation - I+ } \\
\text { (franchissement de côté mains de face, élévation du bassin } \\
\text { proche de l'horizontale) } \\
\text { Damien: Tu ne montes pas assez les fesses! C'est dommage! } \\
\text { Kelly: Mais j'ai peur de glisser! } \\
\text { Damien: Pas si tu repousses bien dans les épaules. }\end{array}$ & \\
\hline $\begin{array}{l}\text { Damien [s'adresse à l'ensemble du groupe]: Pour passer di- } \\
\text { rectement, il faut bien appuyer dans le tramp et monter les } \\
\text { fesses! }\end{array}$ & \\
\hline
\end{tabular}

En tolérant les franchissements de profil, qui correspondent - comme souligné dans l'analyse a priori - à des niveaux de franchissement peu denses en savoir, Damien cherche à amener les élèves à franchir directement. S’il juge ces niveaux intéressants pour travailler l'impulsion et le premier envol, il envisage leurs limites quant à l'évolution vers les niveaux supérieurs comme le montrent les extraits d'entretien du tableau 3 (séance 2, épisode 4). Ses interventions confirment la volonté d'aborder très rapidement la totalité du saut en utilisant les niveaux de franchissement (ici les niveaux 3), auxquels il attribue une fonction motivationnelle et de réussite immédiate. 


\section{Différenciation des interventions de Damien en fonction des réponses spéci- fiques des élèves}

En premier lieu, l'enseignant rapproche le tremplin pour les élèves en difficulté et l'éloigne pour les plus forts (séance 1, épisode 5; séance 2, épisode 5). Cette action, qui vise à ajuster l'aménagement matériel de la tâche aux capacités de chacun, est ensuite confiée aux élèves dès la troisième séance. A partir de la deuxième séance, les interventions de Damien sont plus spécifiques des différents enjeux de savoir et dépendent des réalisations des élèves. Ainsi, au cours des séances 2, 3 et 4, comme le montre l'extrait rapporté dans le tableau 3, Damien donne des indications sur l'impulsion pour les élèves en difficulté, en leur demandant d'«enfoncer le tremplin», d' «appuyer dans le tremplin», de "rebondir jambes tendues dans le tremplin», de «ne pas piétiner avant le tremplin» ou d' «élever le bassin au premier envoli. Dans les entretiens, il explicite ses interventions en exploitant des savoirs appartenant aux registres de la biomécanique et de l'anatomie fonctionnelle, ce que traduisent les extraits suivants:

Je veux qu'ils montent bien dans le tramp car ça conditionne l'énergie restituée pour l'élévation au premier envol (séance 3, épisode 4).

Nassim, là, il a trop de composante horizontale dans sa trajectoire et après, il peut pas bloquer les bras sur le cheval, il se laisse embarquer (séance 4, épisode 5).

Il désigne aussi pour ces élèves des repères auditifs (entendre le bruit de l'action dans le tremplin) ou visuels (regarder le cheval au moment de la course d'élan), afin qu'ils mettent en relation des perceptions (des bruits, des sensations, des focalisations visuelles) avec la qualité du saut effectué. Pour les meilleurs élèves, il intervient sur l'orientation du buste de face, l'action de répulsion des bras, autant de techniques envisagées dans l'entretien (EAC, séance 4) comme conditions nécessaires du redressement.

En mettant l'accent sur la description interne et l'efficacité des actions impliquées dans les différentes phases du saut, Damien exploite un discours imprégné de la technologie formalisée par Carrasco (1975) puis Piard (1982) et mobilise des savoirs issus des registres savants (biomécanique, anatomie fonctionnelle, psycho-physiologie). Pour les quatre séances étudiées, les résultats révèlent que Damien privilégie les retours sur les premières phases du saut pour les élèves les plus en difficulté, en insistant sur les indices perceptifs, alors qu'inversement ses interventions portent davantage sur les phases finales du saut pour les meilleurs élèves.

\section{Topogenèse et mésogenèse au service de la chronogenèse}

L'analyse des gestes d'aide à l'étude montre qu'au plan mésogénétique l'exploitation différenciée des niveaux de franchissement s'accompagne d'interventions verbales adaptées aux réalisations des élèves en lien avec les étapes chronologiques du saut. L'avancée du temps didactique, réalisée globalement pour l'ensemble de la classe, respecte le rythme d'apprentissage des élèves situés aux extrêmes (les plus forts et les plus faibles) grâce à un déplacement plus fin du curseur sur 
les différents niveaux en fonction du temps et des élèves. Si l'enseignant oriente le travail collectif des élèves, il permet à chacun, par un guidage de type individualisé, d'adapter le choix du niveau à ses possibilités. La chronogenèse, matérialisée par les niveaux de franchissement hiérarchisés et relayée par les techniques topogénétiques, est en quelque sorte partagée entre les élèves, qui la gèrent de façon individuelle, et l'enseignant qui la prend en charge de façon globale au sein de la classe ${ }^{15}$.

\section{Résultats: Singularité et enchevêtrement de savoirs multiples}

\section{Les éléments théorico-technologiques des praxéologies activées dans les gestes de l'enseignant}

Ayant décrit les gestes de conception des dispositifs et d'aide à l'étude de Damien, il s'agit ici, en remontant à un premier niveau d'inférence, d'identifier les fondements théorico-technologiques des praxéologies gymniques et didactiques qu'il active.

\section{Les praxéologies gymniques}

Définis d'un point de vue fonctionnel, les niveaux de franchissement hiérarchisés correspondent à la réalisation globale d'une forme de franchissement. Ils prennent en compte l'appréhension des élèves et les logiques liées aux différentes phases du saut. Ce qui préside à la hiérarchisation des niveaux, c'est une conception interactive des objets de savoir, organisée autour du découpage chronologique du saut mais reliée à des paliers adaptatifs. Comme évoqué précédemment, les praxéologies reposent ici sur la technologie gymnique de Goirand (1990, 1998) qui valorise l'activité adaptative des élèves autour d'une définition de la gymnastique scolaire articulant prise de risques et maîtrise du risque. Sur le plan théorique, le mouvement gymnique est analysé dans sa totalité en relation avec l'identification des obstacles émotionnels et techniques.

Mais pour définir les enjeux de savoir ou intervenir sur le dispositif proposé dans l'action conjointe avec les élèves, l'enseignant recourt aussi à une analyse technique du saut, de type chronologique. A cette occasion, les praxéologies gymniques s'appuient sur la modélisation technique issue des travaux de Carrasco (1975), où le mouvement gymnique résulte d'une mobilisation articulaire en relation avec des actions musculaires adéquates dans chaque phase du saut.

Cette double référence à des discours technologiques experts a priori contradictoires relève d'une reconstruction personnelle de l'enseignant, où la prédominance de l'un sur l'autre dépend à la fois des gestes de l'enseignant (de conception ou d'aide à l'étude) et des contraintes chronogénétiques. 


\section{Les praxéologies didactiques}

Les choix gymniques de Damien, valorisant l'engagement moteur et émotionnel, sont étroitement articulés à d'autres enjeux repérables dans les praxéologies didactiques. Ils sont liés à la volonté de maintenir l'investissement des élèves et de créer du sens dans les apprentissages, comme en atteste l'extrait suivant:

J'essaie de [...] redonner du sens et du plaisir aux élèves. [...] Le gamin, il fonctionne parce qu'il a envie de fonctionner, parce qu'il va avoir du plaisir ou parce que ça correspond à la représentation qu'il a de l'activité (entretien post-cycle).

L'enseignant justifie l'importance accordée à cette dynamique motivationnelle par les caractéristiques psycho-affectives, socio-culturelles et relationnelles de ces élèves de ZEP, ce qu'il explique à plusieurs reprises dans les entretiens:

Ce sont des élèves qui malgré tout sont très attachés à la note (EAC séance 1, épisode 8).

Et puis, [...] notamment avec les cas les plus perturbateurs, on sait très bien que le côté prise de risques, ça marchera mieux. A contrario, c'est les élèves les plus scolaires, ceux qui ont le moins de problèmes d'agitation, qui vont faire généralement l'enchaînement le plus correct [...] (entretien ante-cycle).

Beaucoup de ces élèves sont en échec permanent, ils ont accumulé tellement de retard, globalement ils sont agressés tout le temps (entretien post-cycle).

Les diverses techniques didactiques utilisées par Damien consistent à: i) guider de façon collective mais aussi individuelle l'activité des élèves (aide à l'étude); ii) organiser les conditions du travail de tous les élèves (conception et aide à l'étude); iii) adapter les formes de groupement aux objectifs poursuivis en jouant sur des regroupements affinitaires ou par niveaux (organisation du cycle); iv) encourager les élèves en difficulté (aide à l'étude); v) mettre en place des procédures de travail favorisant le respect mutuel, l'autonomie par le travail en ateliers et la gestion des groupes (organisation des séances).

Sur le plan théorique, ces procédures d'enseignement, visant à associer les élèves à leurs apprentissages, s'inscrivent dans une conception constructiviste des apprentissages largement soutenue, par ailleurs, par la doxa institutionnelle. Il nous semble possible de considérer qu'elles renvoient au registre des savoirs savants mobilisés par cet enseignant, même s'il existe à ce sujet des controverses au sein des communautés scientifiques de la recherche en éducation.

\section{Identification des savoirs mobilisés par l'enseignant}

Les savoirs mobilisés in situ par Damien, inférés à partir des blocs théorico-technologiques des praxéologies qu'il active, appartiennent aux registres savants et experts et sont étroitement articulés aux fonctions que leur attribue l'enseignant. Comme nous le développons ci-après, la reconstruction par le chercheur ${ }^{16}$ des savoirs mobilisés par Damien, typifiée par quelques extraits de corpus, met en évidence la pluralité des registres convoqués simultanément, ce que nous interprétons comme un enchâssement des savoirs utiles pour l'action didactique. 
L'exploitation de savoirs issus des champs de la mécanique, de l'anatomie fonctionnelle ou de la psycho-physiologie est souvent associée chez Damien à celle des savoirs techniques, comme l'illustrent les extraits suivants:

Aujourd'hui, [...] l'objectif c'est de toucher le cheval qu'avec un appui bref des mains, [...] en essayant [...] de bien repousser le cheval avec les mains (séance 4, épisode 4).

Le trampoline et le tremplin, ce n'est pas le même type d'impulsion. D'une part, tu as une percussion beaucoup plus rapide, brève sur le tremplin et après avec le cheval, on va essayer d'aller le chercher beaucoup plus loin, alors que le mini-tramp, on cherche à monter. On a une impulsion beaucoup plus verticale (EAC, Séance 2, épisode 4).

Ces savoirs permettent à Damien d'analyser les réalisations gymniques des élèves et interviennent secondairement dans le choix des niveaux de franchissement (tableau 1). Ils sont implicitement mobilisés lors des régulations verbales, comme par exemple lorsque Damien donne des indications individualisées aux élèves lors des séances 3 et 4 ou lorsqu'il analyse les prestations des élèves dans les EAC.

De même, lorsque l'enseignant se réfere à des savoirs relatifs aux théories psychologiques ou aux théories de l'apprentissage moteur, il mobilise conjointement des savoirs ayant trait aux sciences de l'éducation, à des référents didactiques en usage dans l'institution ou relatifs à la gymnastique ${ }^{17}$. Par exemple, Damien justifie le choix des niveaux de franchissement indirects par leur fonction motivationnelle et rassurante (EAC de la séance 2, tableau 3). Ces savoirs concernent les processus d'acquisition, tels que les aspects motivationnels et représentationnels, le contrôle cognitif et affectif de la tâche, la progressivité des apprentissages, l'estimation du risque, l'alternance énergétique des phases de travail, la quantité de travail, la gestion de ses émotions, l'entraide, l'émulation collective. Exploités dans les gestes d'aide à l'étude et dans la partie organisationnelle des leçons, ces savoirs visent à adapter les conditions d'enseignement et le style d'intervention aux profils des élèves. Ils renvoient par ailleurs aux injonctions institutionnelles émanant des programmes de la discipline et des réformes éducatives.

Il ressort de cette étude que les modalités de mobilisation de ces multiples savoirs se caractérisent par leur finesse, leur singularité et leur enchevêtrement dans les pratiques, en relation avec les gestes professionnels et les intentions didactiques de l'enseignant.

\section{Discussion: Une mobilisation de savoirs hétéro- gènes selon des configurations singulières}

Mises en relation des savoirs: un processus de reconstruction particulier

Le contexte d'enseignement en milieu difficile, le style pédagogique de l'enseignant, son expérience professionnelle et les particularités de l'activité gymnique 
marquent le caractère singulier des résultats obtenus. Mais la centration sur la mise au jour de "processus mobilisateurs et organisationnels» (Bru, 2002) permet d'envisager les conditions de leur généralisation, en identifiant les dynamiques sous-tendant l'activation des savoirs.

Dans le cadre de notre étude, l'enchevêtrement des savoirs de référence utilisés relève d'un processus d'emboîtement, qui traduit des glissements voire parfois des raccourcis entre registres experts et savants en fonction des préoccupations inhérentes aux situations ${ }^{18}$. Ce processus d'opérationnalisation ou de spécification est à l'origine d'un «registre de savoirs personnels» (Johsua, 1996), qui renvoie ici à la mise en jeu contextualisée des objets de savoirs au sein des différents dispositifs d'étude. Ces mises en relation entre registres savants et experts relèvent de processus relativement stables visant une utilisation pratique de ces savoirs. S'observent alors au niveau de leur spécification en contexte des "glissements inter-registres» (Brière-Guenoun, 2005) qui se traduisent par exemple dans la forte articulation entre choix didactiques des situations et référence aux aspects motivationnels de l'activité gymnique.

\section{Des savoirs dépendants d'influences institutionnelles}

La référence à une théorie interactive et adaptative des apprentissages associée à une valorisation des aspects motivationnels oriente l'activité d'enseignement de Damien, comme l'a montré la reconstruction des praxéologies didactiques. Mais la manière dont ce professeur exploite, selon un processus d'allers-retours permanents, les divers registres de savoirs est guidée par des valeurs éducatives liées à son lieu d'exercice, parmi lesquelles on peut citer la solidarité, le "vivre ensemble» (entretien post-cycle), l'esprit citoyen, la réussite, l'engagement émotionnel, affectif et collectif. L'extrait suivant l'illustre bien:

Ce qui est essentiel pour moi, dans cet établissement [collège ZEP], c'est le respect des autres. L'EPS va permettre effectivement de vivre des situations ensemble où on a besoin de l'autre, où on est contre l'autre [...]. Donc, les élèves vont, à un moment donné, endosser tous les rôles sociaux [...]. Ici, on a fait le choix de leur faire vivre des activités assez diverses [...] sur lesquelles on accroche bien les élèves (entretien post-cycle).

L'incorporation des fonctionnements et finalités éducatives de l'établissement est notamment révélée par la forte prise en compte du projet d'EPS et s'exprime par exemple dans les relations établies par Damien entre investissement, plaisir des élèves et voies d'entrée dans l'activité ${ }^{19}$. Les options éducatives affichées relèvent aussi de la formation initiale, comme le montre l'extrait suivant: "Certains profs formateurs m'ont marqué, par leur attitude, par les conceptions qu'ils faisaient passer» $[\ldots]$ (entretien post-cycle).

Plus généralement, nous considérons que les appartenances institutionnelles influencent les pratiques d'enseignement des professeurs tout au long de leur carrière, selon des proportions variables en fonction de leur personnalité et de leurs expériences ${ }^{20}$, marquant ainsi, comme l'a montré Garnier (2003) leur «rapport au savoir personnel» (Chevallard, 1989). 


\section{Conclusion}

Le concept de praxéologie, associé à une étude ascendante de l'action didactique conjointe du professeur et des élèves, nous a permis d'identifier l'enchevêtrement des savoirs mobilisés en contexte par un enseignant d'EPS et leur lien direct avec les possibles et les contraintes du système didactique duquel ils émergent. Pour passer des blocs théorico-technologiques des praxéologies aux savoirs, nous avons exploité les différenciations caractérisant les «savoirs de référence» (Johsua, 1996) afin de reconstruire ensuite la dynamique de mobilisation de ces savoirs. Il ressort de cette étude de cas que les références savantes ou expertes ne sont pas évacuées mais incorporées à l'ensemble des rapports institutionnels, officiels et personnels (Chevallard, 1989) que chaque professeur entretient vis-à-vis du savoir à enseigner. Les configurations singulières d'activation des savoirs identifiées dans cette recherche montrent aussi que certaines routines du professeur relèvent de "processus de naturalisation" (Chevallard, 1999) consistant à mettre sous silence les fondements théorico-technologiques des praxéologies. Ces constats plaident pour le développement de recherches engageant une compréhension en profondeur de l'épistémologie pratique des professeurs en tant que déterminant des pratiques didactiques (Amade-Escot, Amans-Passaga \& Montaud, 2010, sous presse; Sensevy, 2007).

\section{Notes}

1 Il est issu d'un travail de recherche qui questionnait les modalités d'exploitation de savoirs par deux enseignants d'ancienneté différente dans le métier. Seuls, sont rapportés ici les résultats concernant l'enseignant chevronné.

2 Le franchissement par redressement est une figure gymnique au saut de cheval qui consiste après une course d'élan à coordonner un premier envol, la pose des mains sur le cheval et un franchissement de l'agrès avec passage des jambes, pour se redresser et se réceptionner sur les pieds.

3 Chevallard (1999) emploie le terme de "praxéologie mathématique» que par extension dans cette recherche, nous généralisons en proposant l'expression "praxéologie disciplinaire» pour désigner celles relevant de la discipline considérée. En EPS nous spécifions cette expression en relation avec l'activité physique et sportive (APS) enseignée en termes de "praxéologie gymnique» (voir Brière-Guenoun, 2005).

4 Précisons que l'enjeu de la recherche dont cet article est extrait était d'identifier les savoirs activés par deux professeurs d'EPS d'expérience contrastée dans la perspective de discuter des dispositifs de formation initiale en STAPS. Nous avons donc retenu les catégorisations en usage dans ces filières universitaires, ce qui explique leur caractère indigène et finalement peu construit.

5 Cette temporalité caractérise l'entrée dans les apprentissages et demeure suffisamment longue pour étudier les évolutions introduites par l'enseignant.

6 Que nous différencions de l'analyse a priori des tâches prescrites (par l'enseignant) mettant en jeu ce thème d'étude.

7 Nous reprenons ici les catégories d'analyse telles que développées dans le cadre théorique.

8 Nous reprenons ici le sens attribué par Chevallard à ce terme, tel qu'il a été défini dans le cadre théorique. 
9 Puisque lors de ces entretiens, l'enseignant est questionné sur l'explicitation, le commentaire, la justification, l'interprétation et les significations qu'il accorde aux situations vécues.

10 Eux-mêmes décomposés en unités d'analyse interactives (UAI), relatives aux données filmées, et en unités thématiques (UT), relatives aux données d'entretien correspondantes.

11 Pour un développement, voir Brière-Guenoun, 2005.

12 Les ateliers désignent des espaces de travail délimités et mis en parallèle: ils renvoient à une organisation pédagogique très courante en EPS.

13 Consistant à organiser successivement dans le temps et l'espace plusieurs figures gymniques.

14 Ce sur quoi nous renseignera notamment l'analyse des gestes d'aide à l'étude, dans les sections suivantes.

15 Voir section relative aux gestes de conception.

16 Reconstruction qui s'est appuyée sur le recoupement d'indices issus des différents corpus, relatifs aux gestes de conception et d'aide à l'étude (voir section méthode).

17 Comme développé dans la section relative aux praxéologies didactiques.

18 Comme l'ont mis en évidence les extraits cités précédemment.

19 Voir section relative aux praxéologies didactiques.

20 L'influence de l'institution "club sportif», soulignée dans la thèse de Garnier (2003) et repérée au cours de cette recherche, existe aussi probablement pour chaque enseignant.

\section{Bibliographie}

Amade-Escot, C. (2005). The Critical Didactic Incidents as a qualitative method of research to analyze the content taught. Journal of Teaching in Physical Education, 24, 127-148.

Amade-Escot, C. (2007). Les savoirs au cour du didactique. In C. Amade-Escot (Éd.), Le Didactique (pp. 11-30). Paris: Revue EPS.

Amade-Escot, C., Amans-Passaga, C. \& Montaud, D. (2010, sous presse). Les savoirs mobilisés dans l'action didactique par les intervenants en activités physiques et sportives: Un entrelacs de processus épistémiques et institutionnels. Sciences de la Société, 76.

Barbier, J. M. (1996). Savoirs théoriques et savoirs d'action. Paris: PUF.

Bouthier, D. \& Durey, A. (1994). Technologie des APS. Revue Impulsions, 1, 95-124.

Brière-Guenoun, F. (2005). De l'observation des pratiques aux connaissances mobilisées par le professeur dans l'interaction didactique. Le cas du franchissement par redressement au saut de cheval en collège. Thèse de Doctorat STAPS, non publiée, Université d'Orléans.

Bru, M. (2002). Pratiques enseignantes: Des recherches à conforter et à développer. Revue française de pédagogie, 138, 63-73.

Carrasco, R. (1975). L'activité du débutant. Paris: Vigot.

Chevallard, Y. (1989). Le concept de rapport au savoir, rapport personnel, rapport institutionnel, rapport officiel. In Actes du séminaire de didactique des mathématiques et de l'informatique (pp. 211-235). Grenoble: LSD Imag et Institut J. Fourrier.

Chevallard, Y. (1992). Concepts fondamentaux de la didactique: Perspectives apportées par une approche anthropologique. Recherche en Didactique des Mathématiques, 12, (1), 73112.

Chevallard, Y. (1997). Familière et problématique, la figure du professeur. Recherche en Didactique des Mathématiques, 17, (3), 17-54.

Chevallard, Y. (1999). L'analyse des pratiques enseignantes en théorie anthropologique du didactique. Recherches en Didactique des Mathématiques, 19, (2), 221-266.

Garnier, A. (2003). Le rapport au savoir du professeur: Entre contrainte et autonomie. Une étude de cas lors d'un cycle d'enseignement de la gymnastique au collège. Thèse de doctorat en sciences de l'éducation, non publiée, Université Paul Sabatier, Toulouse III.

Goirand, P. (1990). Didactique de la gymnastique et éducation physique. In Association des 
enseignants d'EPS (Éd.), Éducation physique et didactique des APS (pp. 74-87). Paris: AEEPS.

Goirand, P. (1998). L'EPS au collège et gymnastique. Paris: INRP.

Johsua, S. (1996). Le concept de transposition didactique n'est-il propre qu'aux mathématiques? In C. Raisky \& M. Caillot (Éd.), Au-delà des didactiques, le didactique. Débats autour des concepts fédérateurs (pp. 61-73). Bruxelles: De Boeck.

Leutenegger, F. (2009). Le temps d'instruire. Approche clinique et expérimentale du didactique ordinaire en mathématique. Berne: Peter Lang

Léziart, Y. (1997). Savoirs savants et transposition didactique en éducation physique et sportive. STAPS, 42, 59-72.

Marsenach, J. (1991). Quelques aspects du fonctionnement de l'enseignement de l'EPS. In J. Marsenach et al. (Éd.), EPS: Quel enseignement? (pp.33-58). Paris: INRP.

Perrenoud, P., Altet, M., Lessard, C. \& Paquay, L. (Ed.). (2008). Conflits de savoirs en formation des enseignants. Entre savoirs issus de la recherche et savoirs issus de l'expérience. Bruxelles: De Boeck.

Piard, C. (1982). Fondements de la gymnastique: Technologie et pédagogie. Paris: Vigot.

Robin, J. F. (2003).Transposition didactique: Le rôle des leaders en gymnastique scolaire. In C. Amade-Escot (Éd.), Didactique de l'éducation physique. État des recherches (pp. 27-48). Paris: Revue EPS.

Schubauer-Leoni, M. L. (2008). La construction de la référence dans l'action conjointe professeur-élève. In N. Wallian, M. P. Poggi \& M. Musard (Éd.), Co-construire des savoirs: Les métiers de l'intervention par les APSA (pp. 67-86). Besançon: PUFC.

Schubauer-Leoni, M. L. \& Leutenegger F. (2002). Expliquer et comprendre dans une approche clinique/expérimentale du didactique ordinaire. In F. Leutenegger \& M. Saada-Robert (Éd.), Expliquer et comprendre en sciences de l'éducation (pp. 227-251). Bruxelles: De Boeck.

Schubauer-Leoni, M. L. et Leutenegger, F. (2005). Une relecture des phénomènes transpositifs à la lumière de la didactique comparée. Revue suisse des sciences de l'éducation, 27, (3), 407429.

Schubauer-Leoni, M. L., Leutenegger, F., Ligozat, F. \& Fluckiger, A. (2007). Un modèle de l'action conjointe professeur-élèves: Les phénomènes didactiques qu'il peut/doit traiter. In G. Sensevy \& A. Mercier (Éd.), Agir ensemble: L'action didactique conjointe du professeur et des élèves (pp.51-91). Rennes: PUR.

Sensevy, G. (2002). Des catégories pour l'analyse de l'action du professeur: Un essai de mise à l'épreuve. In P. Venturini, C. Amade-Escot \& A. Terrisse, Études des pratiques effectives: L'approche des didactiques (pp. 25-46). Grenoble: La pensée sauvage.

Sensevy, G. (2007). Des catégories pour décrire et comprendre l'action didactique. In G. Sensevy \& A. Mercier (Éd.), Agir ensemble: L'action didactique conjointe du professeur et des élèves (pp.13-49). Rennes: PUR.

Sensevy, G. \& Mercier, A. (Éd.), (2007). Agir ensemble:l'action didactique conjointe du professeur et des élèves. Rennes: PUR.

Sensevy, G., Mercier, A. \& Schubauer-Leoni, M. L. (2000). Vers un modèle de l'action didactique du professeur. À propos de la course à 20. Recherche en Didactique des Mathématiques, 20, (3), 263-304.

Serre, J. C. (1984). La danse parmi les autres formes de la motricité. Recherche en danse, 3, 135-156.

Shulman, L. S. (1987). Knowledge and teaching: Foundation for a new reform. Harvard Educational Review, 51, 1-22.

Tardif, M., Lessard, D. \& Lahaye, L. (1991). Les enseignants des ordres d'enseignement primaire et secondaire face aux savoirs. Esquisse d'une problématique du savoir enseignant. Sociologie et société, 23, (1), 55-69. 
Mots clés: Enseignement secondaire I, éducation physique, savoirs d'enseignants; didactique

\section{In-Situ-Analyse der genutzten Kenntnisse eines Sportlehrers in der didaktischen Interaktionen}

\section{Zusammenfassung}

Diese Studie betrifft die von einem Sportlehrer im Unterricht auf der Sekundarstufe I genutzten Kenntnisse. Die Kenntnisse des Lehrers werden anhand einer Analyse seiner Bewegungen betreffend „Konzept der Lerneinrichtungen“ und «Lernhilfe» erfasst. Die verwendeten Methoden, um den Charakter diese Kenntnisse zu ergründen, stützen sich auf eine Kombination von Filmsequenzen mit Gesprächsaufnahmen von Befragungen im Zusammenhang mit der Konfrontation mit dem Filmmaterial. Die Ergebnisse dieser Studie zeigen, dass sich die unterschiedlichen genutzten Kenntnisse des Sportlehrers vor allem an erzieherischen Werten orientieren, die eigenen Überzeugungen des Lehrers darstellen und darüber hinaus die Zugehörigkeit zu den verschiedenen Institutionen, die er im Laufe seiner Karriere durchlaufen hat, spiegeln.

Schlagworte: Sekundarstufe I, Sportunterricht, Lehrerwissen, Didaktik

\section{Le conoscenze mobilizzate da un docente di educazione fisica durante l'interazione didattica: un'analisi situata}

\section{Riassunto}

Questo studio riguarda la conoscenza usata da un docente di educazione fisica nell'insegnamento quotidiano nella scuola media. La conoscenza del docente viene colta attraverso l'analisi delle sue pratiche professionali, ad esempio "progettare un'attività di apprendimento" e "accompagnare l'apprendimento dello studente". Per accedere alla situazionalità della conoscenza del docente, il metodo usato mette in relazione registrazioni video e interviste di "auto confrontazione". I risultati mostrano la mobilitazione di conoscenze orientate a valori educativi, che traducono le convinzioni del docente e al di là dell'appartenenza alle diverse istituzioni attraversate durante la sua carriera.

Parole chiave: Educazione fisica, conoscenze, mobilitazione 
The knowledge mobilised by a physical education teacher during didactic interactions: a situated analysis

\begin{abstract}
This study is about the knowledge used by a Physical Education teacher when teaching gymnastic during everyday lessons in a middle school. Teacher's knowledge is grasped through the analysis of his professional gestures like «designing learning tasks» and "accompanying the students' learning». To access to the situativity of the teacher's knowledge the method links video data and "auto confrontation" interviews. Findings show the diversity of the knowledge used by the teacher, the fact that it is educational value oriented, that it expresses the teacher's self conceptions, and farther along the strong influence of the teacher's institutional adherence.
\end{abstract}

Key words: Middle school, physical education, teacher's knowledge, didactics 